\section{Case Reports in Oncology}

\title{
Osimertinib Did Not Respond to a Pulmonary Adenocarcinoma with Triple Mutations of Epidermal Growth Factor Receptor, G719S, T790M and S768I
}

\author{
Seigo Minami Shouichi Ihara Tsunehiro Tanaka Hideyasu Okada \\ Kazuki Hashimoto Kiyoshi Komuta \\ Department of Respiratory Medicine, Osaka Police Hospital, Osaka, Japan
}

\section{Keywords}

Uncommon epidermal growth factor receptor (EGFR) mutation · Osimertinib · EGFR exon 18 G719S · EGFR exon 20 S768I · EGFR exon 20 T790M

\begin{abstract}
Uncommon epidermal growth factor receptor (EGFR) gene mutations include G719S, T790M and S768I. T790M gatekeeper mutation is the most frequent mechanism of acquired drug resistance to first- and second-generation EGFR-tyrosine kinase inhibitors (TKIs). Osimertinib is a specific EGFR-TKI to overcome T790M resistance mutation. However, owing to a new drug and a rare mutation type, it remains unknown whether osimertinib is effective for acquired S768I. Herein, we reported a 76 year-old woman with pulmonary adenocarcinoma, which had acquired EGFR mutations of S768I and T790M in addition to original G719S after long gefitinib treatment. These mutations were detected in biopsy specimen of liver metastases. During two months of osimertinib, multiple liver metastases progressively enlarged. This case suggested that acquired S768I mutation might be resistant to osimeritinib, despite of co-occurrence of T790M.




\section{Introduction}

Epidermal growth factor receptor (EGFR) gene mutations occur in approximately 40 and $20 \%$ of non-small cell lung cancer in Asian [1] and Caucasian patients [2], respectively. The inframe exon 19 deletions and the exon 21 L858R point mutation are the most frequent and clinically significant EGFR mutations. These two types of mutations are major and common mutations, accounting for approximately $90 \%$ of all EGFR mutations [3]. The remaining $10 \%$ of EGFR mutations is a heterogeneous group of uncommon mutations within exons 18-21, for example, exon $18 \mathrm{G} 718 \mathrm{~S}$, exon $20 \mathrm{~T} 790 \mathrm{M}$ and exon 20 S768I.

Emergence of T790M gatekeeper mutation is the most frequent mechanism of acquired drug resistance to first- and second-generation EGFR-tyrosine kinase inhibitors (TKIs) [4, 5]. Osimertinib, a third-generation EGFR-TKI, is a specific drug to overcome T790M resistance mutation. On the other hand, G719X and S768I point mutations are detected in approximately 3 and 1\% of EGFR mutations, respectively. First generation EGFR-TKIs provided 32 and $42 \%$ of overall response rate for G719X and S768I mutations, respectively [6]. However, the clinical efficacy of osimertinib for these uncommon mutations remains unknown due to a new drug and rare mutation types.

Herein, we reported a case with acquired mutations of S768I and T790M in addition to original G719S after gefitinib monotherapy. Osimertinib was ineffective in this patient.

\section{Case Presentation}

A 76 year-old woman was referred to our hospital three years before because of abnormal chest-X-ray shadow. She was a non-smoker and had diabetes mellitus, hypothyroidism, hypertension, atrial fibrillation and chronic renal failure (estimated glomerular filtration rate $28.4 \mathrm{~mL} / \mathrm{min} / 1.73 \mathrm{~m}^{2}$ at the start of osimertinib). By video-assisted thoracoscopic mediastinal lymph node biopsy, she was diagnosed with adenocarcinoma of the left upper lobe of the lung with bilateral mediastinal lymphadenopathy (c-T2aN3M0 in the 7th TNM classification of lung cancer by the Union for International Cancer Control [UICC]). The peptide nucleic acidlocked nucleic acid PCR clamp method (by LSI medience corporation) detected G719S mutation in EGFR exon 18 from the lymph node sample. One year after concurrent chemo-radiotherapy of cisplatin and vinorelbine, we found a new metastasis in the left adrenal gland. Carboplatin, pemetrexed and bevacizumab followed by maintenance of pemetrexed and bevacizumab achieved partial response. Serum CEA increased from $5.8 \mathrm{ng} / \mathrm{mL}$ (September 2016) up to $19.7 \mathrm{ng} / \mathrm{mL}$ (June 2017), though tumor size remained stable. Thereafter, gefitinib monotherapy started at July 2017, and maintained partial response for 14 months until we found new multiple liver metastases and enlarged left adrenal gland by abdominal plain CT scan (Fig. 1A). Immunohistochemistry of liver tumor biopsy specimens showed positive staining of CK7 and TTF-1, and weak staining of CK20 and Napsin-A. We confirmed metastatic adenocarcinoma and detected T790M and S761I with the original G719S by the Roche cobas EGFR mutation test ver 2.0 (by LSI medience corporation). Osimertinib was initiated in August 2018. Serum CEA temporarily decreased from $448.4 \mathrm{ng} / \mathrm{mL}$ (before osimertinib) to $196.8 \mathrm{ng} / \mathrm{mL}$ (one month after), but rapidly increased to $742.6 \mathrm{ng} / \mathrm{mL}$ (two month after). Plain abdominal CT scan revealed remarkable progression of multiple liver metastases and left adrenal gland (Fig. 1B). At the same time, she suffered from Trousseau syndrome manifested by multiple cerebral infarctions. We discontinued osimertinib, and then introduced atezolizumab. 
Atezolizumab also failed to control the tumor progression. One month after discontinuation of osimertinib, she died at our hospital.

\section{Discussion}

Our case was characteristics of acquired EGFR mutations of T790M and S768I, which was not responsive to osimeritinib at all. In clinical course and response to osimertinib, our case was similar to the previous case reported by Nasu et al, in which osimertinib was unsuccessful for emergence of the same combination of mutations after gefitinib, erlotinib and afatinib [7]. The main lesions were the progression of primary tumor and pleural effusion and the emergence of brain metastases in the previous case [7], while our case presented with the rapid progression of multiple liver metastases and the sudden emergence of Trousseau syndrome. These two cases suggested that acquired S768I may be refractory to osimertinib and accelerate rapid progression, even if it occurs together with T790M.

Regarding EGFR-TKI naïve S768I, afatinib is expected to be more sensitive to this mutation than first-generation EGFR-TKIs. However, osimertinib remains unknown for this treatment line and mutation. There were three U.S. retrospective studies of first-generation EGFRTKI for S768I mutation. First, in a study of compound EGFR mutations at Beth Israel Deaconess Medical Center, among 79 patients with activated EGFR mutations, two (2.5\%) had combined mutations of G719A and S768I. In both of these two patients, first-line erlotinib provided partial response, but only 5-7 months of PFS and 8-10 months of OS from the start of EGFR-TKIs [8]. Second, a collected and retrospective data showed that first-generation EGFRTKI provided 42 and 53\% of overall response rate for S768I single mutation and compound mutations, respectively [6]. Third, in a study of 9 patients with S768I mutation at Mayo Clinic, among 4 patients with metastatic disease treated with erlotinib, one and two patients achieved partial response and stable disease, respectively. The PFS and OS ranged from 3 to 30 months and from 5 to more than 51 months, respectively [9]. In contrast to first-generation EGFR-TKIs, a combined analysis of LUX-Lung 2, 3 and 6 trials, including only eight patients with S768I mutation, showed that afatinib, a second-generation EGFR-TKI, was responsive in all the patients [10]. The discrepancy in response to S768I between the first- and second-generation EGFR-TKIs was consistent with an in vitro sensitivity data using $\mathrm{Ba} / \mathrm{F} 3$ cells. In this in vitro study, afatinib showed very low half maximal inhibitory concentration $\left(\mathrm{IC}_{50}\right.$ ) value to S768I mutation, but both gefitinib and erlotinib had high values of IC $_{50}$. Osimertinib was less sensitive to S768I mutation than afatinib $[6,11]$. A Korean phase II trial showed that osimertinib achieved partial response in 3 patients of 8 with EGFR-TKI naïve S768I mutation (ORR, $37.5 \%$ ) [12]. There was no other clinical data of osimertinib for S768I untreated with an EGFR-TKI.

Osimertinib for S768I mutation after prior therapy with an EGFR-TKI remains unknown. Although little has been reported on acquired S768I, this mutation may be resistant to osimertinib. A pooled analysis of two phase II trials (AURA extension and AURA2) of osimertinib for 697 patients with T790M-positive NSCLC included only 10 patients (1\%) with S768I mutation [13]. A real-world study in Spain (ASTRIS trial) had 5\% of patients with S768I among 132 patients with T790M mutation [14]. However, these two studies have not yet opened efficacy of osimertinib for these specific patients. To our knowledge, there was only a case report of acquired S768I mutation, except for the Japanese case described above. A 43 year-old Italian woman with stage IV adenocarcinoma originally harboring L858R mutation showed an unusual mechanism of gefitinib-resistance, synchronous combination of histological trans- 
formation to squamous cell carcinoma and occurrence of the S768I secondary mutation. Unlike our case, this case did not acquire T790M mutation after 8 months of gefitinib treatment and was not treated with osimertinib [15]. Thus, we should make a careful decision in osimertinib for acquired S768I mutation, even when T790M appears together.

In conclusion, our case showed acquired mutations of S768I and T790M after long term treatment with gefitinib, to which osimertinib did not respond. Osimertinib can not be expected to overcome emergence of S768I mutation together with T790M.

\section{Acknowledgement}

We appreciate Dr. Shouko Ikuta, Dr. Kanako Nishimatsu, Dr. Saori Ikebe (the Department of Respiratory Medicine, Osaka Police Hospital) for their contribution in care and management of this patient.

\section{Statement of Ethics}

The authors have no ethical conflicts to disclose.

\section{Disclosure Statement}

The authors declare that they have no conflicts of interest.

\section{References}

1 Yatabe Y, Kerr KM, Utomo A, Rajadurai P, Tran VK, Du X, et al. EGFR mutation testing practices within the Asia Pacific region: results of a multicenter diagnostic survey. J Thorac Oncol. 2015;10:438-45.

2 Rosell R, Moran T, Queralt C, Porta R, Cardenal F, Camps C, et al.; Spanish Lung Cancer Group. Screening for epidermal growth factor receptor mutations in lung cancer. N Engl J Med. 2009 Sep;361(10):958-67.

3 Shigematsu H, Lin L, Takahashi T, Nomura M, Suzuki M, Wistuba II, et al. Clinical and biological features associated with epidermal growth factor receptor gene mutations in lung cancers. J Natl Cancer Inst. 2005 Mar;97(5):339-46.

4 Sequist LV, Waltman BA, Dias-Santagata D, Digumarthy S, Turke AB, Fidias P, et al. Genotypic and histological evolution of lung cancers acquiring resistance to EGFR inhibitors. Sci Transl Med. 2011 Mar;3(75):75ra26.

5 Tanaka K, Nosaki K, Otsubo K, Azuma K, Sakata S, Ouchi H, et al. Acquisition of the T790M resistance mutation during afatinib treatment in EGFR tyrosine kinase inhibitor-naïve patients with non-small cell lung cancer harboring EGFR mutations. Oncotarget. 2017 Jul;8(40):68123-30.

6 Kobayashi Y, Mitsudomi T. Not all epidermal growth factor receptor mutations in lung cancer are created equal: perspectives for individualized treatment strategy. Cancer Sci. 2016 Sep;107(9):1179-86.

7 Nasu S, Shiroyama T, Morita S, Takata S, Takada H, Masuhiro K, et al. Osimertinib Treatment Was Unsuccessful for Lung Adenocarcinoma with G719S, S768I, and T790M Mutations. Intern Med. 2018 Dec;57(24):3643-5.

8 Kobayashi S, Canepa HM, Bailey AS, Nakayama S, Yamaguchi N, Goldstein MA, et al. Compound EGFR mutations and response to EGFR tyrosine kinase inhibitors. J Thorac Oncol. 2013;8:45-51.

9 Leventakos K, Kipp BR, Rumilla KM, Winters JL, Yi ES, Mansfield AS. S768I Mutation in EGFR in Patients with Lung Cancer. J Thorac Oncol. 2016;11:1798-801.

10 Yang JC, Sequist LV, Geater SL, Tsai CM, Mok TS, Schuler M, et al. Clinical activity of afatinib in patients with advanced non-small-cell lung cancer harbouring uncommon EGFR mutations: a combined post-hoc analysis of LUX-Lung 2, LUX-Lung 3, and LUX-Lung 6. Lancet Oncol. 2015 Jul;16(7):830-8.

11 Banno E, Togashi Y, Nakamura Y, Chiba M, Kobayashi Y, Hayashi H, et al. Sensitivities to various epidermal growth factor receptor-tyrosine kinase inhibitors of uncommon epidermal growth factor receptor mutations 


\section{Case Reports in Oncology}

L861Q and S768I: what is the optimal epidermal growth factor receptor-tyrosine kinase inhibitor? Cancer Sci. 2016 Aug;107(8):1134-40.

12 Ahn MJ, Cho JH, Sun JM, Lee SH, Ahn JS, Park K, et al. An open-label, multicenter, phase II single arm trial of osimertinib in non-small cell lung cancer patients with uncommon EGFR mutation (KCSG-LU15-09). J Clin Oncol. 2018;36(15_suppl):9050.

13 Jenkins S, Chih-Hsin Yang J, Janne PA, Thress KS, Yu K, Hodge R, et al. EGFR Mutation Analysis for Prospective Patient Selection in Two Phase II Registration Studies of Osimertinib. J Thorac Oncol. 2017;12:1247-256.

14 Baz DV, Lario AP, Bueno MM, Sureda BM, Reguard N, Álvarez R, et al. 156P ASTRIS, a real-world study with osimertinib in patients with non-small cell lung cancer (NSCLC) EGFR T790M mutated: characteristics and diagnostic methods used for patients included in Spain. J Thorac Oncol. 2018;13(4):S93-4.

15 Longo L, Mengoli MC, Bertolini F, Bettelli S, Manfredini S, Rossi G. Synchronous occurrence of squamous-cell carcinoma "transformation" and EGFR exon 20 S768I mutation as a novel mechanism of resistance in EGFRmutated lung adenocarcinoma. Lung Cancer. 2017 Jan;103:24-6.
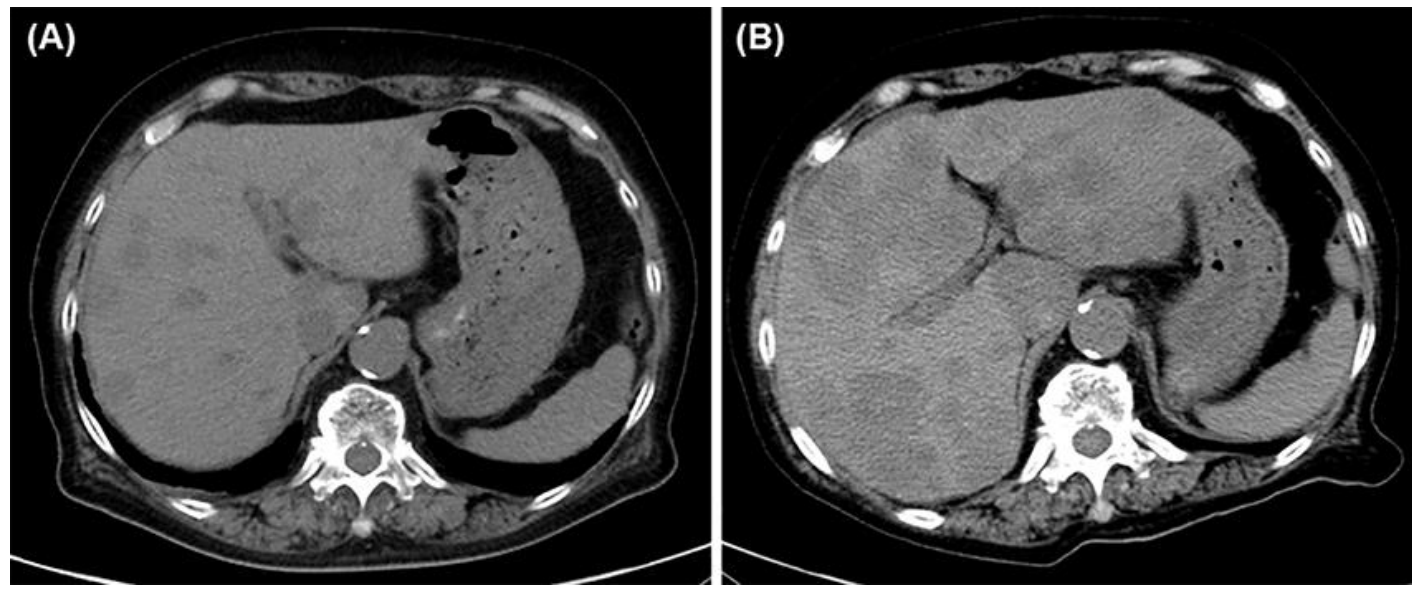

Fig. 1. Plain abdominal CT scan showing multiple liver metastases, (A) before osimertinib, (B) two months after the start of osimertinib. 